\title{
Recht als Mittel der Versöhnung? Die rechtliche Aufarbeitung von Kriegsverbrechen am Beispiel Ruandas Dieter Magsam*
}

\begin{abstract}
In 1994, a long-lasting conflict within Rwandan society escalated into a cruel genocide. Innumerable 'Tutsis' were slaughtered by presidential guards, juvenile militia and others belonging to the ethnic group of the 'Hutus'. Eventually, the FPR overthrew the government and came into power, now confronted with the past and struggling with the question of reconciliation. In choosing to prosecute, the new government has defied the 'culture of impunity' and has thus tried to restore peace. Since regular Ruandan courts, which, in addition to the ICTR, were dealing with crimes committed during the genocide, were overburdened by the number of cases, 'gacacas' (grass-root courts) were established. Whether they are adequate to promote reconciliation has still not been proven.
\end{abstract}

Keywords: Ruanda, Völkermord, Kriegsverbrechen, Gacacagerichte, Versöhnung

\section{Normative Klarstellung: Es geht vor allem um Völkermord}

$\mathrm{D}$ er Titel meines Beitrages kann in seiner Beschränkung auf »Kriegsverbrechen« zu Missverständnissen Anlass geben, weil der Begriff »Kriegsverbrechen« in vielfältiger, teils widersprüchlicher Weise gebraucht wird. Insbesondere bleibt unklar, ob damit auch die Verbrechen gegen die Menschlichkeit und des Völkermordes erfasst werden sollen, wie dies die London International Assembly im Hinblick auf das Statut des Internationalen Militärgerichtshofes empfahl, oder ob damit nur solche Tathandlungen gemeint sind, die in einem funktionalen Zusammenhang mit dem bewaffneten Konflikt stehen. ${ }^{1}$ Die Protagonisten des ruandischen Völkermordes an der als Tutsi definierten Bevölkerungsgruppe werden noch heute nicht müde, die Tötung von 800.000 bis einer Million Menschen im zweiten Quartal 1994 als »funktional « im Hinblick auf die Lösung eines bewaffneten Konflikts zu bezeichnen. ${ }^{2}$ Etwas salopp ausgedrückt: Krieg ist Krieg, beim Hobeln fallen Späne, und wenn einzelne Kombattanten damals durch Anordnungen und/oder Aktionen etwas zu weit gegangen sein sollten, dann haben sie das selbst zu verantworten.

Die Weltöffentlichkeit und insbesondere die Vereinten Nationen haben viel zu lange damit gewartet, die Vorgänge in Ruanda als

* Dieter Magsam, Strafverteidiger, Hamburg; z.Z. Leiter des GTZ-Justizprojektes in Kigali/Ruanda.

1 Vgl. dazu nur Werle,»Völkerstrafrecht «, Tübingen 2003, S.295 f. (Fn.2) und S. 325.

2 S. etwa die Verteidigungslinie im sog. »Militärprozess« (./. Bagosora u.a) vor dem Internationalen Strafgerichtshof für Ruanda in Arusha www.ictr. org. Ein entsprechendes Dokument der Exilregierung vom Juni 1995, die sich nach ihrem Abgang aus Kigali zeitweise in Bukavu (Ostkongo) festgesetzt hatte und mit dessen Verbreitung man der Weltöffentlichkeit gegenüber das Image von Völkermördern abstreifen wollte, befindet sich in meinem Besitz. Die Geschichte wollte es, dass der damalige Autor noch bis vor kurzem als »mandataire d'état « als einer der fähigsten Juristen der gegenwärtigen Regierung galt. Das änderte sich mit einer Vorladung als Experte vor den ICTR. Seine ehemaligen Mitstreiter hatten seine Anhörung dazu verlangt, dass eventuelle damalige Exzesse nur als Kollateralschaden im Zusammenhang mit der bewaffneten Rückkehr der FPR aus Uganda nach Ruanda anzusehen wären. Der Autor berief sich auf damalige Informationmängel, widerrief schriftlich gegenüber dem Gericht sein damaliges Pamphlet und hat sich so den Auftritt in Arusha erspart. Allerdings arbeitet er auch nicht mehr im Justizministerium. Das hätte man sich in den fünfziger Jahren auch bei manchem deutschen Ministerialbeamten gewünscht.
Völkermord zu bezeichnen. Man wollte, falls man nicht ohnehin mit dem Habyarimana-Regime und seinen Nachfolgern, also den Tätern, zusammenarbeitete, den damit verbundenen internationalen Handlungspflichten entgehen. ${ }^{3}$ Zudem saß Anfang 1994 das Völkermordregime als nichtständiges Mitglied selbst im Sicherheitsrat. Scherrer wirft diese Unfähigkeit und den Unwillen, den Völkermord als das zu bezeichnen, was er war, auch den internationalen Hilfsorganisationen vor, wenn er ausführt:

\begin{abstract}
»...Die MitarbeiterInnen der Hilfswerke übernahmen zuerst die Sprachregelung der Massenmörder, dann gaben sie ihnen Jobs. NGO Vertreter sprachen immerzu von einem Krieg, von ,vor dem Krieg' und ,nach dem Krieg'. Die meisten zivilen Helfer waren nicht in der Lage, zwischen Krieg und Genozid zu unterscheiden bzw. Opfer und Täter zu trennen... «4
\end{abstract}

Die Gefahr der begrifflichen Verharmlosung der Verbrechen, die in Ruanda vor zehn Jahren geschehen sind, wird dadurch vermieden, dass wir allgemein und unmissverständlich trotz des obigen Titels nachfolgend von der strafrechtlichen Aufarbeitung des Völkermordes, von Verbrechen gegen die Menschlichkeit und erst dann von Kriegsverbrechen begangen an Ruandern und Ruanderinnen sprechen. Diese Klarstellung erst, die zudem die von den Mördern konstruierte rassistische Zielgruppenbildung nicht zum Ausgangspunkt der eigenen Analyse macht, öffnet den Blick für die Dimension der Konflikte, die heute auf den verschiedensten juristischen Verfahrensebenen schwelen und uns der Antwort auf die Ausgangsfrage, ob diese Verfahren als »Mittel der Versöhnung« dienen, näher bringen.

3 Vgl. dazu insbesondere L. Melvern, »A People betrayed - The role of the West in Rwanda's genocide«, 4. Aufl., London/Berlin 2004; dies.: Ruanda - Der Völkermord und die Beteiligung der westlichen Welt, Kreuzlingen/ München 2004.

4 Scherrer, Christian, »Ethnisierung und Völkermord in Zentralafrika, Bürgerkrieg in Burundi und die Rolle der Weltgemeinschaft«, Frankfurt/ New York 1997, S. 113 


\section{Der Kampf gegen die »Kultur der Straflosigkeit«}

Im Umgang mit der Vergangenheit unterscheidet sich Ruanda von Südafrika. Weltweit hat die fast zeitgleich gefeierte Befreiung von dem Apartheidregime den Blick auf die ruandischen Besonderheiten getrübt. Die dort eingerichtete Wahrheits- und Versöhnungskommission mit ihrem weitgehenden Strafverzicht, u.a. eine Folge der Verhandlungslösung beim Machtübergang, galt als Prototyp eines afrikanischen Ansatzes für Versöhnung. ${ }^{5}$ Nicht nur wegen der gemessen an Südafrika riesigen Zahl von Tätern und Opfern hat sich Ruanda in bewusstem Gegensatz dazu entschieden, »die Kultur der Straflosigkeit« zu beenden. Auch die Tatsache, dass der Völkermord durch einen militärischen Sieg beendet wurde, reicht als Erklärung nicht aus. Entscheidend war, dass diese Unkultur zum Gründungsfundus der Ruandischen Republik gehörte und seit 30 Jahren immer wieder zu ungesühnten völkermordnahen Massakern an der als Tutsi bezeichneten Bevölkerungsgruppe geführt hatte. Sie hatte sich wie Mehltau in den Zeiten der 1. und 2. Republik bis hin zur versuchten »Endlösung « im Jahre 1994 über das Land gelegt und war auch in der unmittelbaren Nachgenozidsituation, in der die Grundsatzentscheidungen über das weitere Vorgehen zu fällen waren, nicht aus den Köpfen und - viel gefährlicher - aus den Bauchregionen verschwunden. Das Wiederaufleben der rassistischen und von den Kolonialmächten konstruierten »Hamitentheorie«, wonach es sich bei »den Tutsi« um eine aus Nordostafrika eingewanderte Rasse handle, die das »Hutu-Volk« auf dem Gebiet Ruandas unterworfen habe, musste verhindert werden. Denn diese besagt aus der Sicht der Mörder nichts anderes, als dass die als »Tutsi « bezeichnete Bevölkerung im Gebiet der Großen Seen prinzipiell nichts zu suchen und dort wenn überhaupt nur ein Lebensrecht auf Bewährung hätte. ${ }^{6}$ Ruandas politische Realität war jahrzehntelang dadurch gekennzeichnet, dass dort nicht nur etwa $15 \%$ der Bevölkerung qua Verfassung diskriminiert wurden, sondern auch auf Abruf lebten. Rückkehrversuche der zwischen 1959 und 1964 vertriebenen Generation, bestehend aus Menschen, die man durchgängig als Mitglieder und Steigbügelhalter der abgeschafften Tutsinen-Monarchie denunzierte, wurden ebenso mit Massakern an den im Land verbliebenen 15\% beantwortet, wie Rückkehrversuche der zweiten Flüchtlingsgeneration ab Oktober 1990, die wie der gegenwärtige Präsident und seine Bewegungspartei FPR mit der Monarchie erklärtermaßen nichts zu tun hatte. Die drohende Rückkehr der etwa 500.000

$5 \quad$ Ruandische Zyniker weisen bitter darauf hin, dass es an der amerikanischen Ostküste mittlerweile mehr Dissertationen über die »Versöhnungsarbeit « bzw. »-bereitschaft «, der Afrikaner' gibt als in Ruanda Studenten. Die amerikanische Filmerin Anne Aghion, die seit Jahren das Weiterleben auf einem Hügel in der Nähe von Gitarama filmerisch begleitet, zeigt in ihrem letzten Film (2004) zwei ältere Frauen, die überlebt haben und sich über die ständig nach Versöhnung fragenden Bleichgesichter lachend wundern. Dabei sollte man wissen, dass Lachen in Ruanda die gegensätzlichsten Empfindungen begleitet.

6 Die Literatur hierzu ist mittlerweile unüberschaubar. Aus jüngerer Zeit hierzu insbesondere Nigel Eltringham, »Accounting for Horror: PostGenocide Debates in Rwanda«, London 2004. Dieses Werk hat nicht nur den Vorteil, das reden über das angebliche Vorhandensein ethnischer oder rassischer Gruppen auf ruandischem Boden in seiner Wirkung auf den Genozid und dessen Aufarbeitung in den Mittelpunkt zu stellen. Es liefert auch eine Bibliographie, in der kein Standardwerk zu der zu verneinenden Frage »Handelt es sich bei Tutsi und Hutu überhaupt um Ethnien?« fehlt Zum Punkt Versöhnung: Wer vorschnell als die zu versöhnenden Parteien oder Individuen die Kategorien »Hutu « und »Tutsi« verwendet, tappt nach Eltringham genau in die Falle, die die Völkermörder aufgestellt haben. grenznahen ruandischen Flüchtlinge ${ }^{7}$ in ihr Heimatland, die nach dem zeitgleichen Wegfall der Blockkonfrontation auch der Westen befürwortete, gefährdete das Monopol des inneren Zirkels auf Ressourcenzugang (u.a. Entwicklungshilfe) und Macht. Ein relevanter Teil dieser Rückkehrwilligen war politisch organisiert. Wie schon 1963/64 wurde die bedrohte Macht durch zwei Strategien, die beide die als Tutsi gelabelten ruandischen Bevölkerungsteile zum Spielball hatte, verteidigt. Die erste Strategie bestand darin, die FPR zu erpressen: Wenn ihr zurückkommt, findet ihr keine Tutsi mehr vor. ${ }^{8}$ Die zweite bestand darin, die Interessen der Elite dadurch zu verteidigen, dass man sie als solche der restlichen $85 \%$ verbrämte (das Boot ist voll; der Tutsi-Nachbar ist Teil der 5. Kolonne der Angreifer, die Dich verjagen wollen etc.), einen möglichst großen Teil der bäuerlichen Massen in die Tötungsaktionen einzubinden versuchte und so die »Hutu-Nation « unter Führung eines zentralistischen Apparates - aber eben nicht nur unter Druck - in einer Art bluttriefendem Initiationsritual ${ }^{9}$ neu begründete bzw. verschweißte.

Dieser auf die Zerstörung einer eher als Rasse denn als Ethnie konstruierten ${ }^{10}$ Gruppe gerichtete Mechanismus, der ohne die lange vorher begonnene, durch Straflosigkeit des Tötens bezeugte und von großen Teilen der Bevölkerung akzeptierte Entrechtung der Opfer nicht hätte funktionieren können, musste in seiner Dialektik aus Zwang und Freiwilligkeit in einem auf individuelle Schuldfeststellung und entsprechende Konsequenzen angelegten Verfahren aufgeklärt werden, wenn Wiederholungen vermieden und ein einigermaßen sicheres und friedliches Weiterleben erreicht werden sollten. Im Gegensatz zu Deutschland nach dem Sieg über den Faschismus gibt es in Ruanda nämlich eine relevante Anzahl Überlebender und weiterer potentieller Opfer. Das jedenfalls waren Mitte der neunziger Jahre die Vorstellungen der neuen ruandischen Regierung, die aus den Kräften hervorgegangen war, die den Völkermord beendet hatten bzw. nicht an ihm beteiligt waren. ${ }^{11}$ Wir haben diese Entscheidung, über den Weg strafrechtlicher Verfolgung (»ohne Gerechtigkeit keine Versöhnung «) auch breitester Bevölkerungsteile zum inneren

7 Zahlen und Aufschlüsselung der Flüchtlinge nach Ländern in der Region der Großen Seen bei M. Mamdani: »When Victims Become Killers Colonialism, Nativism, and the Genocide in Rwanda«, Kampala/Oxford/ Capetown 2001.

8 Wegen dieser infamen Strategie hatte schon im Dezember 1963 der ruandische König im Exil bewaffnete Rückkehrversuche zu verbieten versucht; vgl. Filip Reyntjens, »Pouvoir et Droit au Rwanda. Droit public et évolution politique 1916-1973«, Bruxelles 1985, S. 469. Wie bei allen Geiselnahmen gibt es hier einen objektiven Interessengegensatz zwischen Innen und Außen. Auch wenn sie das Rückkehrrecht der Flüchtlinge der 2.Generation nicht bestritten, haben viele im Lande lebende Ruander mit dem Passeintrag» Tutsi« auf den Oktoberangriff 1990 der FPR schockiert reagiert - »das ist unser Ende«; die sich innerhalb Ruandas neu formierende Opposition arbeitete zunächst mit der FPR zusammen, verurteilte deren militärische Lösungsversuche aber mitunter auch als terroristische Akte, die den Demokratisierungsprozess innerhalb des Landes zurückwürfen und den Hardlinern in die Hände spielten, vgl. hierzu insb. J. Bertand, Le Rwanda, le piége de l'histoire. L'échec de l'opposition démocratique (20002004 ) , Paris 2000.

9 Siehe zu diesem Erklärungsversuch des Unerklärbaren E.K. Ohlendorf, "Zehn Jahre Genozid - Der Völkermord in Rwanda: Gruppenidentität und Gewalt« in: Institut für Afrikakunde (Hrsg): »Afrika im Blickpunkt«, März 2004.

10 Vgl. hierzu die berechtigte Kritik von Eltringham an den Akayezu- und Kayishema. Entscheidungen des ICTR; a.a.O. S.31 ff.

11 Siehe auch F. Digneffe et J. Fierens( eds.), "Justice et gacaca. L'expérience rwandaise et le génocide«, Namur 2003, S.48 ff (»La reconnaissance par la communauté internationale et par le Rwanda lui-même de ce qu'un genocide avait été commis rendit impérieuse la nécessité de réagir de manière extrèmement forte et claire «) 
Frieden zu gelangen und dem südafrikanischen Beispiel nicht $\mathrm{zu}$ folgen, zu respektieren.

Auf die damit verbundenen Schwierigkeiten, Ungerechtigkeiten und Gefahren komme ich nachstehend zu sprechen. Sie treten bei der Internationalen Gerichtsbarkeit in Arusha ebenso auf wie bei dem Versuch der strafrechtlichen Aufarbeitung in Ruanda selbst.

\section{Die Auswirkungen der Internationalen Strafgerichtsbarkeit}

Zunächst soll auf die Internationale Strafgerichtsbarkeit zu Ruanda eingegangen werden. Der im tansanischen Arusha am Verhandlungsort der gebrochenen Verträge errichtete Internationale Strafgerichtshof für Ruanda (ICTR) ist befugt,

»Personen, die für schwere Verstöße gegen das humanitäre Völkerrecht, welche zwischen dem 1. Januar 1994 und dem 31. Dezember 1994 im Hoheitsgebiet von Ruanda begangen wurden, sowie ruandische Staatsangehörige, die für derartige Verstöße verantwortlich sind, welche in demselben Zeitraum im Hoheitsgebiet von Nachbarstaaten begangen wurden, ... zu verfolgen.« (Art.1 des Statuts)

Zum humanitären Völkerrecht zählt das Statut Völkermord (Art. 2), Verbrechen gegen die Menschlichkeit (Art. 3) und schwere Verstöße gegen den Genfer Abkommen vom 12. August 1949 gemeinsamen Artikel 3 über den Schutz von Kriegsopfern und das dazu gehörende Zusatzprotokoll vom 8. Juni 1977 (Art. 4). Von der Kompetenz des ICTR wären damit auch Straftaten von Soldaten und ihren Befehlshabern gedeckt, die den Völkermord beendet haben, soweit sie in das Jahr 1994 fallen und den Tatbestand des Verbrechens gegen die Menschlichkeit oder auch des Art. 4 des Statuts - etwa Kollektivstrafen, Hinrichtungen ohne vorausgehendes Urteil oder Plünderungen - erfüllen. Bisher ist ein solches Verfahren in Arusha noch nicht anhängig gemacht worden ${ }^{12}$, obwohl sich massive Hinweise auf solche Verbrechen nicht nur für die Zeit von Oktober 1990 bis Ende $1993^{13}$, sondern auch für das Jahr 1994, das in das Mandat des ICTR fällt, finden. ${ }^{14}$ Da das Mandat für Ermittlungen Ende 2004 ausläuft und das letzte erstinstanzliche Urteil Ende 2008 fallen muss, ist mit einem Prozess, der Verbrechen der siegreichen Militärs betrifft, auch nicht mehr zu rechnen. Für die gegenwärtige Situation in Ruanda wäre der Vorteil einer hierauf gerichteten öffentlichen gerichtlichen Aufklärung nicht zu überschätzen. Dies gilt für die ruandische Justiz selbst - dazu weiter unten - in verstärktem Maße. Könnte doch wie etwa beim Jugoslawiengerichtshof durch das Erkennen auf allen Seiten begangener Straftaten der Eindruck einer einseitigen Siegerjustiz vermieden und die Macht des Rechts gestärkt werden. Ein plastisches Beispiel: Ein Leutnant der früheren Armee hat hier in Ruanda die eigene Beteiligung an Verbrechen

12 Die von abgehalfterten ruandischen und europäischen Extremisten, aber auch regierungsnahen Kreisen (etwa in Frankreich) zur eigenen Entlastung immer noch gestreute Theorie des Doppelgenozids ist absurd.

13 Schon das Arusha-Protokoll vom 18. August 1992 sah in Artikel 16 die Bildung einer internationalen Untersuchungskommission vor, die Menschenrechtsverletzungen beider Seiten untersuchen sollte. Vollständiger Text bei Imbleau Schabas, »Introduction au droit rwandais«, Cowansville (Quebec) 1999, S.304 ff.

14 FIDH und HRW unter der Redaktion von Alison des Forges, »Kein Zeuge darf überleben - der Genozid in Ruanda«, Hamburg 2002, S. 823-860. eingeräumt und wurde nach langen Untersuchungshaftjahren im Hinblick auf die mit dem Geständnis verbundene geringere Straferwartung vorläufig entlassen. Er sollte und wollte zudem in Arusha in dem gegen Simba u.a. laufenden Verfahren als Belastungszeuge aussagen und wurde aus bisher gerichtlich nicht ermittelten Gründen kurz vor seiner Abreise nach Arusha ermordet. ${ }^{15}$ Ein Bekannter des Ermordeten, der selbst über acht Jahre nachweisbar unschuldig in Untersuchungshaft saß, berichtete mir, der Getötete habe auch ein Dossier über Straftaten der FPR in Arusha abliefern wollen. Er sei deswegen - davon sei er überzeugt - von der Regierung umgebracht worden. Die offizielle Version, bevor überhaupt ein Richter die Akte gesehen hat, lautet natürlich genau gegensätzlich - und die Kommunikationsblockade ist perfekt. Jedes unaufgeklärte Verbrechen wird so zur Projektionsfläche dessen, was man aufgrund eigener leidvoller Erfahrungen anderen zutraut.

Wäre die internationale Strafgerichtsbarkeit glaubhaft auch mit Straftaten der oben beschriebenen Art befasst (und wäre auch in Ruanda selbst der abwartende Respekt vor der Justiz verbreiteter), ließe sich eine die Versöhnung erschwerende Legendenbildung und die Kultivierung der jeweils eigenen Opfermentalität vermeiden. Bekanntlich stand im Oktober 2003 die Verlängerung des Amtes für die damalige Chefanklägerin Carla del Ponte an, die hatte verlauten lassen, dass sie in einer weiteren Amtszeit ebensolche Verbrechen untersuchen wollte. Kigali hatte mit seiner daraufhin verstärkten Polemik gegen den ICTR und Frau del Ponte mit amerikanischer Unterstützung Erfolg. ${ }^{16}$

Abgesehen hiervon sind die Ausstrahlungen der Spruchtätigkeit in Arusha auf die gesellschaftlichen Prozesse in Ruanda eher gering. Allenfalls die im Verhältnis zu den ruandischen Lebensbedingungen luxuriösen Haftbedingungen in Arusha und die dort anfallenden Verfahrenskosten geben Anlass zu populistischer Polemik. Das alles soll sich mit einer gerade begonnenen Informationskampagne des ICTR ändern. Ändern kann sich die relative Bedeutungslosigkeit auch, wenn wegen des zeitlich begrenzten Mandats des Arushatribunals einzelne Verfahren gegen die erste Riege der Angeklagten nach Kigali abgegeben werden. Es ist abzusehen, dass wegen der u.a. den höheren Verfahrensstandards geschuldeten geringen Urteilszahlen (z.Zt. 22 bei zwei Freisprüchen) nicht alle der an sich vorgesehenen Verfahren dort beendet werden können. Vorverhandlungen laufen, und der ruandischen Regierung ist bewusst, dass die gewünschte Übernahme dieser Verfahren an den Ort des Geschehens nur um den Preis des Verzichtes auf die Vollstreckung der Todesstrafe zu haben ist. Man stünde dann vor dem widersinnigen Befund, dass die vor den nationalen Gerichten Ruandas angeklagte zweite Garde der Völkermörder immer noch der Todesstrafe ins Auge sieht, während die Haupttäter davon verschont blieben. Eine generelle Abschaffung der Todesstrafe - letztlich ein Symbol dafür, dass die Versöhnungsoption offen gehalten wird - ist leider kurzfristig nicht zu erwarten. Wenn auch seit 1998 die für die nationale Gerichtsbarkeit in bestimmtem Fällen obli-

\footnotetext{
15 Hirondelle News Agency 20.10.2004 »Prosecution Witness Assassinated in Rwanda $\ll$.

16 Eine Würdigung des sonstigen Auftretens Frau del Pontes in Arusha, falls sie sich dort überhaupt einmal sehen ließ, ist mit diesem bedauerlichen Aspekt ihres Abdankens nicht verbunden.
} 
gatorischen Todesurteile aufgrund präsidialer Verfügung zur Zeit nicht vollstreckt werden, wird mit der Aufrechterhaltung der absoluten Strafe dem Ansehen der Justiz auf die Dauer jedoch kein Gefallen getan: Hängt die Vollstreckung gesetzeskonformer Gerichtsurteile regelmäßig von der Exekutive ab, wird letztere zumindest in den Augen der Bevölkerung über das Gesetz gestellt und die Etablierung eines rechtsstaatlichen Bewusstseins erschwert.

Noch eine abschließende Bemerkung zu der Weigerung der ruandischen Regierung, die Untersuchung von auf ihrer Seite vorliegender Straftaten im Zusammenhang mit der Rückkehr nach Ruanda und der Befreiung des Landes zuzulassen: Möglicherweise fiele es ihr leichter, Verbrechen der ihr zuzurechnenden politischen und militärischen Kräfte einzuräumen oder Material für Untersuchungen zur Verfügung zu stellen und damit Versöhnungshürden abzubauen, wenn internationale Kräfte mit gutem Beispiel vorangingen. Ich denke da an Frankreich und stelle die Frage, ob deutsche Entwicklungspolitik im good-governance-Bereich möglicherweise auch darin bestehen könnte, positiv auf die europäischen Partner einzuwirken. Genauso machen wir uns in Ruanda unglaubwürdig, wenn wir zu Guantanamo schweigen ${ }^{17}$ und in Afrika menschenwürdige Haftbedingungen einfordern, die auch in Ruanda nicht sehr verbreitet sind. Eine als »technisch « bezeichnete Zusammenarbeit gerät hier offensichtlich an die Grenzen ihres Mandats. An einer intelligenten Lösung dieses Glaubwürdigkeitsproblems, das für unsere konkrete Tätigkeit im Bereich verantwortlicher Regierungsführung auch und gerade eines der Effektivität darstellt, sollten wir arbeiten.

\section{Versuch der juristischen Aufarbeitung in Ruanda}

Die nationalen ruandischen Versuche, die oben beschriebene Grundsatzentscheidung zur Strafverfolgung umzusetzen, begannen als Projekt der gesamten Regierung im Jahre 1996 nach längeren Konsultationen und mehreren internationalen Konferenzen. Es gab damals etwa 70.000 der Sache nach Untersuchungsgefangene, die ernährt werden mussten und über deren Zukunft zu befinden war. Als »loi organique«, die den Kontext des Völkermordes umfassend umsetzte, das heißt vor allem auch Prozessfragen und einfachere Verstöße wie Diebstähle und Sachbeschädigungen regeln wollte, die im programmatischen Völkermordzusammenhang begangen wurden, aber eigentlich keine Völkermordhandlungen darstellten, erging das Gesetz vom 30. August 1996. Entgegenstehende einfachgesetzliche Regelungen traten zurück, nicht aber das Militärstraf- und -strafprozessrecht. Alle von Militärs begangenen Straftaten sollten vor Militärgerichten verhandelt werden. Im Übrigen führte die »loi organique « eine Kategorisierung ein, die die Beschuldigten in vier Gruppen von Haupttätern des Völkermordes und Verbrechen gegen die Menschlichkeit bis hinunter zu einfachen Dieben einteilte. Prozessual zuständig sollten neu zu besetzende Spezialkammern bei den erstinstanzlichen Gerichten sein. Eine Spezialkammer der »Cour Suprême «

17 Vgl. dazu U. Wesel, »Die Konjunktur der Menschenrechte. Ein Ausflug
nach Guantanamo«, in »Kursbuch« Heft 155 (Neue Rechtsordnungen), Berlin, März 2004, S. 28 ff. war insgesamt für die Durchführung und Überwachung verantwortlich. Durch rechtzeitige glaubhafte Geständnisse, die von Reue und Vergebungswunsch getragen auch Mittäter benennen, war eine erhebliche Strafreduzierung möglich. Hiervon versprach man sich neben verfahrensökonomischen Vorteilen auch Wahrheitsfindung und die Aussicht auf gesellschaftliche Versöhnung, insbesondere, wenn der Wunsch nach Vergebung auf Opferseite akzeptiert wird. Die Zahlen der von 1997 bis 2000 auf dieser Basis verurteilten Personen stieg von 346 (1997), 928 (1998), 1318 (1999) auf 2458 im Jahre 2000. Aufgrund weiterer Verhaftungen stieg parallel dazu jedoch die Zahl der Untersuchungsgefangenen stetig an, bevor sie Ende 2002 etwa 130.000 erreichte. Der erste Ansatz konnte absehbar nicht durchgehalten werden und führte zu einem Lösungsversuch, der mit dem Namen »gacaca « verbunden ist. Gewählte Laienrichter sollten für die 2.-4. Kategorie zuständig sein, während die ordentlichen Gerichte die Zuständigkeit für die bereits bei ihnen anhängigen Verfahren behielten und darüber hinaus zukünftig nur noch über Angeklagte der 1. Kategorie richten sollten. ${ }^{18}$ Man versprach sich dadurch eine Entlastung der ordentlichen Justiz, die bis Ende 2004 etwa 8.000 Urteile gefällt hat, davon etwa 20\% Freisprüche. Aussagen über den Versöhnungseffekt dieser Verfahren wären rein spekulativ. Zwar wurden Angeklagte neben den Todesund Haftstrafen zu zum Teil nach ruandischen Verhältnissen immensen Entschädigungszahlungen verurteilt; deren Zahlung steht aber in den Sternen.

Es gibt bis heute entgegen verbreiteter Vorstellung noch kein einziges Urteil der Gacacagerichte. Über die Eignung dieser Verfahren, zur Versöhnung beizutragen, lässt sich ehrlicherweise schon deswegen nur spekulieren. Von den 2002 gewählten etwa 250.000 Dorfrichterinnen sind im Juni 2004 nach einer Gesetzesänderung, die u.a. aus prozessökonomischen Gründen die 3. Kategorie abschaffte und den dort erfassten Personenkreis der 2. zuschlug, etwa 75.000 wieder ausgeschieden. Nach den Erfahrungen von Pilotgacacas, die bis zur Kategorisierung vorgedrungen sind und etwa 10\% der Taten erfassten, sowie aus der Auswertung der von dem Justizprojekt der gtz in Zusammenarbeit mit der Generalstaatsanwaltschaft erstellten Datenbank ergibt sich folgendes Bild:

- Die Zahl der vor den ordentlichen Gerichten zu verhandelnden Strafsachen der 1. Kategorie wird sich auf etwa 50.000 belaufen.

- Die Zahl der Beschuldigten insgesamt liegt zwischen 500.000 und 600.000 Personen, davon wahrscheinlich etwa 400.000 in der 2. Kategorie.

- Geständnisse im Zusammenhang mit ca. 225.000 Sexualstraftaten liegen kaum vor.

- Die Laienrichter verfügen nicht über Befragungsmethoden, die die Beurteilung der Glaubwürdigkeit von Geständnissen erlauben. Geständnisse sind oft mechanisch und räumen nur das ein, was ohnehin feststeht.

- Der allein zur Verfügung stehende Personalbeweis ist nicht nur wegen des Zeitablaufes, sondern auch deswe-

$\overline{18}$ Ebenso wie das Gesetz vom August 1996 erstreckte sich das Gacaca-Gesetz vom Dezember 2001 auf die zwischen dem 1.10.1990 und dem 31.12.1994 begangenen Verbrechen des Völkermords und gegen die Menschlichkeit und der in diesem Zusammenhang begangenen auch einfacheren Straftaten, nicht aber auf von Militärs begangene Delikte. 
gen fragwürdig, weil aus Opferperspektive - jedenfalls zum Tatgeschehen - wenig zu berichten ist. Den Opfern persönlich nahestehende Personen direkt am Tatort haben selten überlebt und Belastungszeugen - nicht selten vom Hörensagen - sind gerade deswegen noch am Leben, weil sie wegen Tatortferne nichts sehen konnten.

- Sollte es anders sein, sind die Schwierigkeiten für Zeugen und Zeuginnen, in öffentlicher Verhandlung als eine der wenigen überlebenden Personen inmitten der Familien von mutmaßlichen Tätern, die z.T. noch im Gefängnis sitzen und bei festem Zeugenauftritt auch bleiben würden, immens.

- Es sind jetzt schon zehn Jahre mit immerwährenden Befragungen und ohne Resultate vergangen. Ein Gesetz über die Entschädigung der Opfer gibt es nicht. Sie erhalten lediglich freie ärztliche Versorgung und kostenlosen Schulbesuch für die Kinder.

- Es gibt immer noch etwa 60.000 Untersuchungsinhaftierte, davon unter Berücksichtigung der bisherigen Freispruchsquote bei den ordentlichen Gerichten wahrscheinlich über 10.000 Unschuldige. ${ }^{19}$

Um die Gefängnisse zu leeren, wurden Anfang 2003 aufgrund eines Präsidialerlasses etwa 22.000 Personen entlassen. Neben besonders Alten und besonders Jungen handelte es sich um solche Gefangene, die Geständnisse abgelegt hatten, dadurch in einen niedrigeren Strafrahmen gerutscht sind und jetzt in Freiheit die Urteilsphase abwarten. Viele Überlebende auf den Hügeln haben mit Unverständnis reagiert. Andere wiederum stellen die Notwendigkeit des Miteinander-AuskommenMüssens in den Vordergrund.

In einem jüngst erschienenen Bericht einer Parlamentskommission werden erstmals die bei Gacaca bisher aufgetretenen Probleme erwähnt und die Schuld dafür »progenozidären « oder »sektiererischen « NGOs in die Schuhe geschoben, anstatt die strukturellen Probleme offen einzuräumen und nach besten Kräften zu beheben.

- Ein umfassender Zeugenschutz ist schlicht nicht möglich. Wenn insbesondere in der Provinz Gikongoro die Bedrängung von Belastungszeugen bis hin zur Ermordung zunimmt, kann die Antwort nicht darin bestehen, regierungskritische NGOs dafür verantwortlich zu machen.

- Der ruandische Staat reagiert auch wegen der ethnizistisch aufgeladenen Zuspitzung der Situation in den Nachbarländern Kongo und Burundi mit einer Verschärfung des innenpolitischen Kurses. Wo der repressionsfreie innerruandische Dialog zur Aufklärung dessen unumgänglich wäre, was 1990 bis 1994 im Lande geschehen ist und wie die Geschehnisse auf den Einzelnen gewirkt haben, wird dieser zunehmend mit Hilfe eines an Unbestimmtheit nicht $\mathrm{zu}$ überbietenden Gesetzes aus dem Jahre 2001 unterdrückt. ${ }^{20}$

- Da - wenn überhaupt - einzelne die damalige Zeit betreffende Militärstraftaten vor Militärgerichten nicht öffentlich verhandelt werden, entsteht auf Angeklagtenseite

19 Einen guten Überblick über den Stand der Gacacas, der letzten Gesetzesänderungen gibt www.penalreform.org.

20 Loi $\mathrm{N}^{\circ} 4712001$ du 18/12/2001 Portant Repression des Crimes de Discrimination et Pratique du Sectarisme. zudem der weitverbreitete Eindruck, dass die »Kultur der Straflosigkeit« für Militärs weiterhin gelte.

- Ohne der FPR, die immerhin den Völkermord beendet hat, eine Mitschuld daran zuzuweisen, wird sie an einer Diskussion nicht vorbeikommen, ob sie sich zwischen 1994 und der Beendigung des Völkermordes stets an den Interessen der im Lande lebenden »Geiseln « orientiert hat. Viele Überlebende des Völkermordes sehen die FPR als neue Machtelite, der das Schicksal der schon immer in Ruanda Lebenden gleichgültig war und - siehe Entschädigungsfrage - ist. Beide Gruppen sind Opfer - auch die zurückgekehrten anglophon gewordenen Flüchtlinge, die ihre Jugend in den Camps in Tansania, Uganda usw. vergeudet haben. Diese »concurrence des victimes « (Chaumont) schwelt, wird aber zum Teil unter Strafdrohungen (sectarisme) unterdrückt.

- Hinzu kommen die vielen unschuldig Inhaftierten und ihre Familien.

\section{Ausblick}

Alle diese Menschen - auch die Regierungsmitglieder und ihre Familien - müssen in einer Umgebung leben, in der jeder achte Erwachsene in irgendeiner Form am Völkermord beteiligt war. Das friedliche Zusammenleben zu ermöglichen, ohne dass sich Gruppeninteressen absondern und bedrohlich aufstauen, scheint mir die wichtigste konfliktpräventive Aufgabe der Gegenwart in Ruanda zu sein. Die den neuen ruandischen Staat repräsentierenden Kräfte sind selbst Teil dieser Konflikt-Gemengelage, denen eine unparteiische Führung der staatlichen Angelegenheiten nicht leicht fällt. Bei dieser fortdauernden Diversität und Massivität der Zerklüftungen kann ich persönlich mit dem Begriff der Versöhnung wenig anfangen. Da jeder bisherige Völkermord eine staatlich organisierte Veranstaltung war, spricht viel dafür, dass die Kontrolle der staatlichen Macht durch den Aufbau eines rechtsstaatlichen Systems ein vorrangiges, wenn auch nur langfristig zu erreichendes Ziel der Entwicklungszusammenarbeit in Ruanda zu sein hat. Andererseits stellt die starke staatliche und militärische Präsenz in fast allen gesellschaftlichen Bereichen Ruandas unbestreitbar auch einen Sicherheitsfaktor da. Durch schrittweise, aber fühlbare juristische Einzäunung der Exekutive wird den Überlebenden und Traumatisierten, aber auch den unschuldig Inhaftierten und jetzt Freigelassenen Sicherheit vor dem Staat in Aussicht gestellt und gleichzeitig ein Rahmen geschaffen, innerhalb dessen die unterschiedlichen Opferperspektiven, aber auch politischen Interessen offener formuliert werden können. Versöhnung als individuelle Leistung eines jeden Opfers gegenüber einem bestimmten Täter kann man in diesem Kontext nur respektvoll herbeihoffen.

Als justiz- bzw. strafrechtsähnlicher Versuch, Wahrheitsfindung und Versöhnung zu organisieren, ist Gacaca zwar noch nicht gescheitert, aber die Skepsis nimmt zu. Es besteht die Gefahr, dass wegen des Fehlens rechtsstaatlicher Mindeststandards - wofür es viele objektive Gründe gibt - letztlich keines der Ziele erreicht wird. Immerhin aber wird allein durch die landeswei- 
te Mobilisierung und die Organisation der »Rasengerichte ${ }^{21}$ symbolisch deutlich, dass noch lange gemeinsam über die Entstehung und Verarbeitung der Katastrophe geredet und nachgedacht werden soll und dass man - im Prinzip jedenfalls - eine Kollektivschuld der Nicht-Opfer verneint. Hinter dem Versöhnungs- und Einheitsdiskurs der herrschenden politischmilitärischen Kräfte sollte man indessen sehr genau nach spezifischen Interessen (und totalitaristischer Ausbeutung des Genozidbonus) schauen. Das unbestreitbare Verdienst, einen Völkermord beendet zu haben, und die Furcht vor einem Aufleben neuer Konflikte können auf Dauer demokratische Defizite nicht entschuldigen. ${ }^{22}$ Wo - wie in jeder Gesellschaft - Konflikte schwelen, provoziert die strafrechtlich durchgesetzte Beschwörung von Einheit und Versöhnung oft das Gegenteil. Demokratie und Rechtsstaat entzaubern das »Volksmärchen« der Versöhnung, oder, wie Alain Finkielkraut es in seiner Analyse des Barbie-Prozesses für eine andere Post-Genozidphase formuliert hat:

»....doch leider behängen wir den Antinazismus mit dem totalitären Phantasma der offenen Herzen und des Glücks der Verschmelzung. Dem Trauma der homogenen Volksgemeinschaft aus Blut und Boden setzen wir die »übertriebene Nähe einer gleichmacherischen Brüderlichkeit entgegen. Ganz so als wäre nichts geschehen, als hätte keine Katastrophe unsere Epoche zu einer Epoche der Trauer gemacht, sinkt die Nacht der Idylle wieder auf uns herab. Die Liebe entthront Polemos, das Gefühl füllt die Zwischenräume

21 Die ja traditionell gerade das Gegenteil staatlicher Gerichte waren, nämlich echte grass-root settings darstellten.

22 Vgl. hierzu das Diskussionspapier von P. Uvin, »Wake up! Some Policy Proposals for the International Community in Rwanda«, June 2003 (»...improved rule of law is probably the socio-political change with the highest pay-off for Rwanda's future«). der Differenz aus und ersetzt die antagonistische Äußerung von Meinungen durch die lyrische Vereinigung von Personen...In einer scheinbaren Versöhnung mit den Idealen der Demokratie verdunkelt sich die Politik...« ${ }^{23}$

Auf die Eingangsfrage bezogen heißt das: Konflikte sind normal und bedürfen an sich keiner besonderen Behandlung in Form vom Versöhnungskampagnen. Der Rechtsstaat und die Politik sind schlicht dazu da, die friedliche Austragung dieser Konflikte zu ermöglichen. Ruanda war und ist kein »failing state«, im Gegenteil: Gerade die effektive, europäischen Vorbildern nachempfundene Verwaltungsstruktur erlaubt es heute, innerhalb von drei Tagen ein nationales Verbot des Urinierens in der Öffentlichkeit und der Benutzung von Plastiktüten zu etablieren. Diese Struktur war Voraussetzung des Genozids. Bei einem Staat, dessen Größe der Belgiens und dessen Einwohnerzahl der des OLG-Bezirkes Hamm entspricht und in dem es im Gegensatz zu vielen anderen Ländern Afrikas keine lokalen Rechtstraditionen (mehr) gibt, können mit der Etablierung landesweit wirksamer rechtsstaatlicher Strukturen die Wiederholung der Katastrophe vermieden und jedenfalls die Rahmenbedingungen für individuelle TäterOpfer-Verständigung geschaffen werden. Das Beste, was man gegenwärtig über die Gacaca-Verfahren sagen kann, ist, dass sie eine conditio sine qua non für die innerruandische Verständigung darstellen und trotz ihrer Mängel alternativlos sind. Nach den ersten Urteilen, die in etwa einem Jahr zu erwarten sind, und den Erfahrungen mit weiteren vorläufigen Freilassungen bis dahin wird neu darüber nachzudenken sein.

23 A. Finkielkraut, »Die vergebliche Erinnerung - Verbrechen gegen die Menschheit«, Berlin 1989, S. 87.

\section{Bundeswehr-Modell »2025«}

\section{Kommission »Europäische Sicherheit und Zukunft der Bundeswehr«am IFSH}

\section{Friedenspolitische Grundprämisse}

Jede europäische Gemeinsame Außen- und Sicherheitspolitik muss zuerst und vor allem völkerrechtlich sanktionierte Friedenspolitik, eine Politik der zivilen Instrumente sein. Dem Primat der zivilen Krisenprävention ist durch eine signifikante Umschichtung der Mittel zugunsten nichtmilitärischer Sicherheitskräfte, die für die Gefahren und Risiken der Zukunft von größerer Relevanz sind, Rechnung zu tragen. Nur damit kann das Leitbild der Europäischen Union als »Friedensmacht» Glaubwürdigkeit gewinnen.

\section{Gesellschaftspolitische Prämissen}

2.1 In Europa existiert seit geraumer Zeit ein eindeutiger Trend weg von der allgemeinen Wehrpflicht, hin zu Freiwilligenstreitkräften. Auch die Bundeswehr praktiziert längst keine allgemeine Wehrpflicht mehr, sondern nur noch eine Art Fassadenwehrpflicht: Wenn von 250.000 Soldatinnen und Soldaten nur noch 30.000, also zwölf Prozent, echte Grundwehrdienstleistende sein sollen, kann nicht mehr ernsthaft von Wehrpflichtarmee gesprochen werden. 220.000 Militärangehörige werden zukünftig Freiwillige - Freiwillig zusätzlichen Wehrdienst 